OPEN ACCESS

Edited by:

Lu Yang,

Sichuan University, China

Reviewed by:

Matthew Pierre Deek, Johns Hopkins Medicine,

United States

Carlo Terrone,

San Martino Hospital (IRCCS), Italy

*Correspondence:

Paul Sargos

p.sargos@bordeaux.unicancer.fr

Specialty section: This article was submitted to Genitourinary Oncology, a section of the journa

Frontiers in Oncology

Received: 14 May 2021 Accepted: 05 July 2021

Published: 26 July 2021

Citation:

Giraud N, Buy X, Vuong N-S,

Gaston R, Cazeau A-L,

Catena $V$, Palussiere $J$, Roubaud $G$ and Sargos P (2021) Single-Center Experience of Focal

Thermo-Ablative Therapy After

Pelvic Radiotherapy for In-Field Prostate Cancer Oligo-Recurrence.

Front. Oncol. 11:709779.

doi: 10.3389/fonc.2021.709779

\section{Single-Center Experience of Focal Thermo-Ablative Therapy After Pelvic Radiotherapy for In-Field Prostate Cancer Oligo-Recurrence}

\author{
Nicolas Giraud ${ }^{1}$, Xavier Buy ${ }^{2}$, Nam-Son Vuong ${ }^{3}$, Richard Gaston ${ }^{3}$, Anne-Laure Cazeau ${ }^{4}$, \\ Vittorio Catena ${ }^{2}$, Jean Palussiere ${ }^{2}$, Guilhem Roubaud ${ }^{5}$ and Paul Sargos ${ }^{{ }^{*}}$ \\ ${ }^{1}$ Radiation Oncology Department, Institut Bergonié, Bordeaux, France, ${ }^{2}$ Oncologic Imaging Department, Institut Bergonié, \\ Bordeaux, France, ${ }^{3}$ Urology Department, Clinique Saint Augustin, Bordeaux, France, ${ }^{4}$ Nuclear Medicine Department, Institut \\ Bergonié, Bordeaux, France, ${ }^{5}$ Medical Oncology Department, Institut Bergonié, Bordeaux, France
}

Purpose: In-field prostate cancer (PCa) oligo-recurrence after pelvic radiotherapy is a challenging situation for which metastasis-directed treatments may be beneficial, but options for focal therapies are scarce.

Methods: We retrospectively reviewed data for patients with three or less in-field oligorecurrent nodal, bone and/or locally recurrent (prostate, seminal vesicles, or prostatic bed) PCa lesions after radiation therapy, identified with molecular imaging (PET and/or MRI) and treated by focal ablative therapy (cryotherapy or radiofrequency) at the Institut Bergonié between 2012 and 2020. Chosen endpoints were the post-procedure PSA response (partially defined as a $>50 \%$ reduction, complete as a PSA $<0.05 \mathrm{ng} / \mathrm{ml}$ ), progression-free survival (PFS) defined as either a biochemical relapse (defined as a rise $>25 \%$ of the Nadir and above $2 \mathrm{ng} / \mathrm{mll}$ ), radiological relapse (on any imaging technique), decision of treatment modification (hormonotherapy initiation or line change) or death, and tolerance.

Results: Forty-three patients were included. Diagnostic imaging was mostly $18 \mathrm{~F}-C h o l i n e$ positron emission tomography/computerized tomography (PET/CT) $(75.0 \%)$, prostate specific membrane antigen (PSMA) PET/CT (9.1\%) or a combination of pelvic magnetic resonance imaging (MRI), CT, and $99 \mathrm{mTc}$-bone scintigraphy (11.4\%). PSA response was observed in $41.9 \%$ patients (partial in $30.3 \%$, complete in $11.6 \%$ ). In the hormonesensitive exclusive focal ablation group $(n=31)$, partial and complete PSA responses were 32.3 and $12.9 \%$ respectively. Early local control (absence of visible residual active target) on the post-procedure imaging was achieved with $87.5 \%$ success. After a median followup of 30 months (IQR 13.3-56.8), the median PFS was 9 months overall (95\% Cl, 6-17), and 17 months (95\% Cl, 11-NA) for PSA responders. Complications occurred in 11.4\% patients, with only one grade IIIb Dindo-Clavien event (uretral stenosis requiring endoscopic uretrotomy). 


\begin{abstract}
Conclusion: In PCa patients showing in-field oligo-recurrence after pelvic radiotherapy, focal ablative treatment is a feasible option, possibly delaying a systemic treatment initiation or modification. These invasive strategies should preferably be performed in expert centers and discussed along other available focal strategies in multidisciplinary meetings.
\end{abstract}

Keywords: prostate cancer, focal therapy, cryotherapy, oligo-recurrence, interventional radiology, radiation therapy

\section{INTRODUCTION}

Prostate cancer (PCa) ranks among the leading diagnosed cancers and causes of male cancer deaths worldwide, with an estimated 1,276,000 new cancer cases and 359,000 deaths in 2018 , a number expected to grow in the upcoming years due to the growth and aging of the population (1). Despite recent advances in the metastatic setting, the number of systemic agents remains limited, especially once castration-resistant status is acquired. The role of local therapies, once restricted to localized disease with curative intent or palliative purposes, is gaining importance and has even shown overall survival benefits when treating newly diagnosed low metastatic burden prostate cancer with radiation therapy (RT) to the prostate in addition to the standard systemic treatment $(2,3)$.

The concept of oligo-metastatic disease, originating in Hellman and Weichselbaum theories over 20 years ago, showcases growing interest, notably with the development of more accurate imaging modalities, precise focal treatments like stereotactic body radiation therapy (SBRT), and more conservative surgery procedures $(4,5)$. In $\mathrm{PCa}$, led by advances in terms of imaging with the successive appearance of more sensitive radiotracers in $18 \mathrm{~F}$-sodium fluoride (NaF), 18F-Choline, and 68Ga-prostate-specific membrane antigen (PSMA) positron emission tomography/computed tomography (PET/CT), as well as emerging therapies offering prolonged survival, oligo-metastatic disease is increasingly diagnosed. The goal is to treat locally and aggressively every visible location with curative intent for cancers harboring a small number of metastatic lesions, classically less than five, possibly reflecting less aggressive cancers with better prognosis (6). Recently, a few phase II and III trials have shown the benefit of such strategies in terms of progression-free survival (PFS) and overall survival when used as consolidation or in addition to the systemic standard of care, for various neoplasms (7-9).

In $\mathrm{PCa}$, it is theorized that oligo-metastatic evolutions may account for a peculiar form of prostatic disease intermediary between localized and widespread metastatic disease, with slower growth and less aggressive phenotypes, amenable to metastasisdirected therapies (MDTs) (10). One of the goals is to delay the instauration or modification of a systemic treatment (androgen deprivation therapy (ADT) possibly combined with chemotherapy or next generation hormonal therapy), especially for slowly evolving diseases, to avoid toxicities and gain time before the initiation of a new systemic line. This idea is being explored in ongoing or recently presented phase II-III trials, either with purely pelvic oligorecurrent lesions (excluding patients with history of pelvic RT) in
OLIGOPELVIS 2 and STORM (NCT03569241) (11), or distant metastatic oligo-recurrence with the PCS IX (NCT02685397), PRESTO, STOMP, POPSTAR, POSTCARD, and ORIOLE trials (12-16); MDT being mostly SBRT or surgery in these trials.

Nevertheless, in case of in-field relapses after RT, options may be limited and defining the optimal strategy can be challenging. Salvage therapeutic options may include surgery, high-intensity focused ultrasound (HIFU), or re-irradiation (brachytherapy or SBRT). Thermo-ablative procedures are another viable option, historically mostly used for the salvage treatment of locally recurrent $\mathrm{PCa}$, but can also be used to treat nodal or bone lesions especially when not amenable to other MDTs.

In this study, we aimed to retrospectively assess the oncological and toxicity outcomes after thermo-ablative therapy for in-field oligo-recurrent $\mathrm{PCa}$ ( $\leq 3$ bone, nodal and/or locally recurrent lesions) in our French experienced single center.

\section{MATERIALS AND METHODS}

\section{Patient Selection and Collected Variables}

All patients harboring $\mathrm{PCa}$ who received thermo-ablative procedures for metastatic prostate cancer between January 2012 and December 2020 in our center were retrospectively reviewed. Inclusion criteria were patients with oligo-recurrence eligible for MDT to all visible lesions on imaging (PET-CT or a combination of CT/MRI/bone scintigraphy) delivered with curative intent. Patients without history of RT, with lesions not located inside a previous RT field (inside a previous planning target volume with curative intent, according to contemporary recommendations (17-19)), or with non-adenocarcinoma histology were also excluded. Patients treated with a palliative pain-relief objective (palliative treatment in poly-metastatic patients), or with $>3$ lesions were also excluded. All treatment decisions must had been validated in multidisciplinary concertation meetings. Institutional Review Board approval was obtained.

Variables of interest were extracted from individual patient medical records, including age of patients at the time of procedure, initial PCa characteristics [Gleason score and ISUP group, cTNM, and pTNM in case of surgery, initial prostate serum antigen (PSA), D'Amico risk classification], and previous treatment sequence (surgery, radiation therapy, prior ADT). Limited lymphadenectomy included the obturator chain bilaterally, while extended lymphadenectomy involved bilateral chains: obturator, external-, internal-, common iliac and 
presacral (20). The latest pre-procedure PSA results were congregated in order to assess the PSA doubling-time (PSADT) according to the MSKCC nomogram (21). The number and localization of treated lesions, diagnostic imaging modality as well as the thermo-ablative technique (radiofrequency or cryoablation) and eventual use of concomitant systemic treatment were compiled.

During follow-up, every 3 months for 6 months then every 6 months, acute and late toxicities, dates of biochemical, radiological progressions, and date of death were collected, as well as the date of systemic treatment initiation or modification (including ADT and date of castration-resistant systemic line initiation) and date of new focal treatment. Date of last follow-up was defined as the last available consultation date or date of death.

\section{Thermo-Ablative Procedures}

Procedures were performed by two interventional radiologists with several years of experience in percutaneous thermal ablation of various tumors such as liver, kidney, lung, and musculoskeletal tumors. To offer a better comfort to the patients and as prone position was often required to reach the tumor, general anesthesia was preferred.
Prostatic bed or endopelvic soft tissue ablation was performed with cryoablation, using a last generation argon-based cryoablation machine (Visual Ice, Boston-Scientific, USA). The number and type of cryoprobes (17 gauge IceRod or IceSphere) varied, depending on tumor size and location. All endopelvic procedures were performed under CT guidance to achieve optimal control of the ice ball and surrounding at-risk organs (Figure 1). For all interventions, a double $10 \mathrm{~min}$ freezing cycle was applied.

Pelvic bone metastases were treated with radiofrequency ablation (Cool-tip, Medtronic, USA) using a single 17 gauge electrode. A 6 to $10 \mathrm{~min}$ ablation time was applied to achieve power rolloff. The goal was to reach a temperature plateau $>65^{\circ} \mathrm{C}$ at the tip of the electrode.

Cryoablation was preferred for soft tissue lesions offering a precise control of the ice flow and a real time visualization in these structures to protect highly vulnerable surrounding structures (e.g., sciatic nerve branches or urinary meatus). In opposition, radiofrequency was favored for bone lesions because of a faster procedure, its lower cost, and the lesser concern about surrounding structures as well as a mediocre visualization of the cryoablation ice flow in bone densities. To protect surrounding organs such as rectum, ureters, bladder, or nerves, various thermal protection

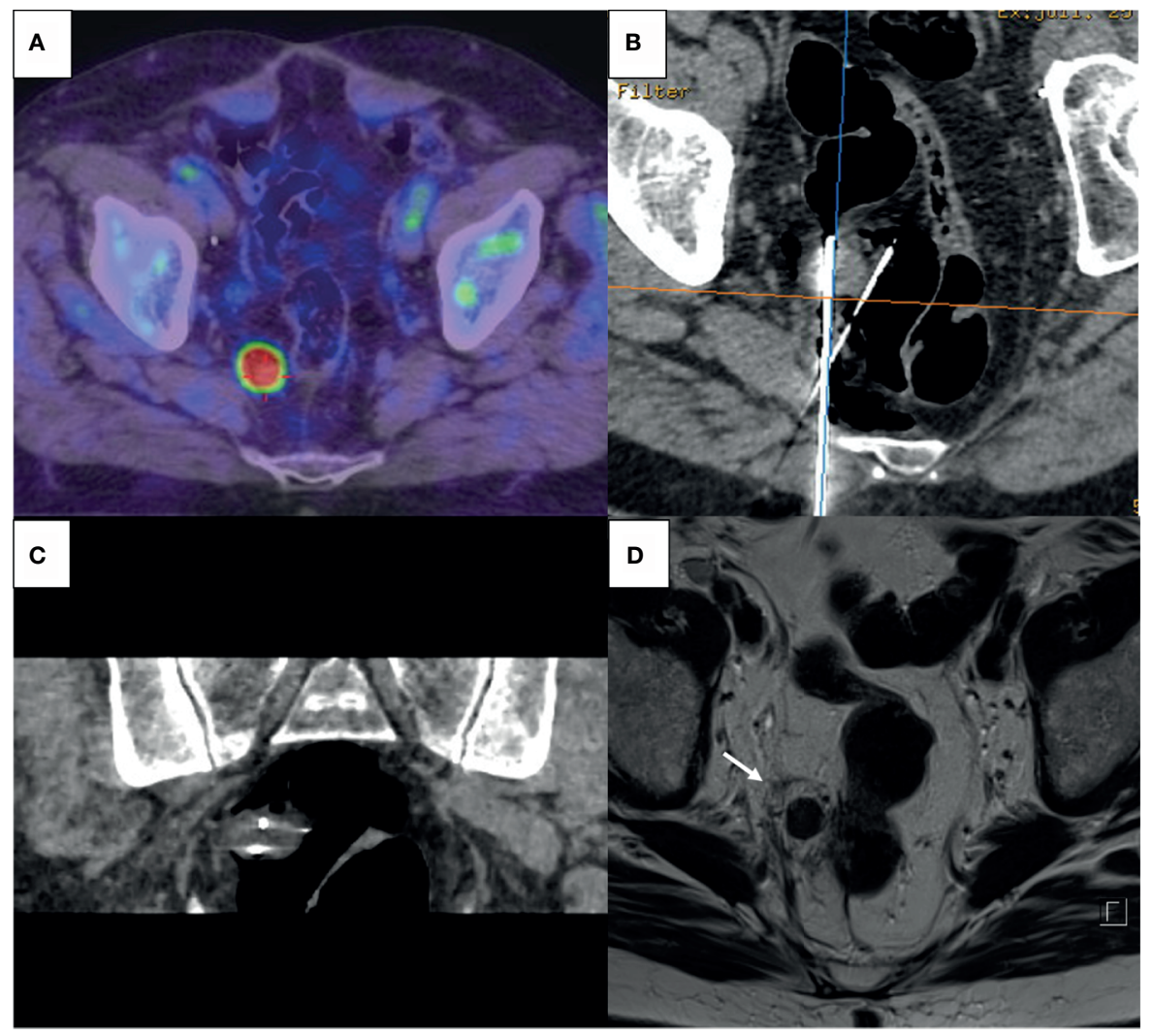

FIGURE 1 | (A) Pre-procedure PET-Choline showing a $2 \mathrm{~cm}$ hypermetabolic lymph node abutting the rectal wall. (B, C) CT-guided percutaneous cryoablation with axial view (B) and coronal reconstruction (C); two cryoprobes were inserted into the target and $\mathrm{CO}_{2}$ insufflation via a 22-gauge spinal needle was performed to achieve rectal wall displacement and isolation. (D) T2-weighted MRI after 6 weeks showing complete necrosis of the nodule with surrounding halo of cytosteatonecrosis (arrow); absence of complication on the rectal wall. 
techniques were used (22). Organ displacement or insulation was performed with hydrodissection or $\mathrm{CO}_{2}$ dissection. For tumors abutting major nerves, additional thermocouple was inserted to achieve continuous focal temperature monitoring.

\section{Outcomes}

The primary endpoint was the PSA response, defined as partial ( $>50 \%$ decline from pre-procedure PSA) or complete (PSA $<0.05$ $\mathrm{ng} / \mathrm{ml}$ post-procedure $)(23,24)$. PFS was defined, similarly to the ORIOLE trial (15), as failure occurring during follow-up of either a biochemical progression (PSA increase of $>25 \%$ of the Nadir and $>2$ $\mathrm{ng} / \mathrm{ml}$ ), ADT initiation for any reason, clinical or radiological progression [according to RECIST (25)], or death. In our center, ADT initiation was routinely discussed in a multidisciplinary setting and motivated based on the evidence of radiological metastatic evolution after biochemical relapse per the Phoenix guidelines (PSA $\geq 2 \mathrm{ng} / \mathrm{ml}+$ Nadir) or a combination of characteristics according to the European guidelines (PSA-DT $<6-12$ months and Gleason $>7$, ISUP grade $>3$ ) if no metastatic lesion was highlighted $(26,27)$.

A post-procedure local evaluation (preferably by MRI) was almost systematically performed during the following first months in order to assess the early local control (defined as the absence of visible local residual active disease) and absence of procedure-related complication.

Acute ( $\leq 3$ months) and distant toxicities during follow-up were also collected and graded according to the Dindo-Clavien classification (28).

\section{Statistical Analysis}

Statistical analyses were performed to compare subgroups using Student's t-test if applicable or Wilcoxon tests for quantitative variables, and Fisher exact tests for qualitative variables.

Log rank tests and Kaplan-Meier curves were used to assess survival outcomes. P-value of $<.05$ was considered significant. All statistical tests were computed using RStudio (v1.3.959).

\section{RESULTS}

\section{Characteristics of Patients}

Between January 2012 and December 2020, 105 focal thermoablative procedures (cryoablation or radiofrequency) were performed for 79 patients with PCa in our center. Among these 79 patients, twenty were treated for palliative purposes (pain relief of bone metastases), seven for extra-pelvic lesions and were thus excluded. Nine other patients were excluded for various reasons (no pelvic RT or lesions outside the previous field of treatment for five patients, concomitant bladder and prostate primaries for one patient, more than three lesions for two patients, undifferentiated histology for one patient). Detailed flowchart can be visualized in Figure 2.

In the end, our cohort consisted of 43 patients treated for infield pelvic oligo-recurrent relapses, with a total number of 49 lesions treated. To be noted is that one patient had three nodal lesions, one in the pelvis treated by cryotherapy and two others outside the previous radiation fields treated by RT. Baseline characteristics of these 43 patients are presented in Table 1.
In this population, one patient was already treated for castrationresistant PCa with enzalutamide, the focal treatment goal aiming to avoid a change of systemic line, considered as an "oligo-progressive disease" (29). Likewise, four patients were undergoing total androgen blockade, the focal approach hoping to postpone a castration-resistant systemic first line. Finally, six patients started short ADT and one already under ADT received the addition of bicalutamide concomitant to the focal treatment.

Considering our ADT-free hormone-sensitive population of 31 patients, the median age was 72 years (range, 51-81), with patients presenting mostly high initial d'Amico risk scores (93.5\%). Pre-procedure PSA was $2.48 \mathrm{ng} / \mathrm{ml}$ (range, 0.43-14.5) and pre-procedure calculated PSA-DT was 7.6 months (range, 1.4-55.1). Thirty-four lesions were treated by thermo-ablative therapy among which six were pelvic bone lesions (pubic rami, symphysis), twelve were nodal targets and sixteen were (in place or post-surgical) prostate or seminal vesicle recurrences.

\section{Diagnostic Modality and Ablative Procedure}

Diagnostic imaging consisted in PET-Choline for thirty-three patients (76.7\%), PET-PSMA for four patients (9.3\%), PET-NaF for one patient $(2.3 \%)$, and a combination of MRI, CT, and bone scintigraphy for five patients $(11.6 \%)$.

Focal thermo-ablative procedure was RF for 10 lesions (20.4\%) and cryotherapy for 39 lesions (79.6\%). Only one lesion, situated on the pubic symphysis, received an additional thermo-ablative treatment due to its size and the persistence of visible disease on the post-procedure MRI. Among the overall population, respective median and mean maximum diameters of the treated lesions were 12 and $15.2 \mathrm{~mm}$ (range, 6-57); 11 and $12.3 \mathrm{~mm}$ (range, 6-35) in the ADT-free cohort.

\section{Outcomes}

Among the overall population of 43 patients, PSA response was observed in $41.9 \%(n=18)$ : partial in $30.3 \%(n=13)$ and complete in $11.6 \%(n=5)$ patients. The post-procedure local evaluation was performed in $90.9 \%$ of patients $(n=40)$, with a median delay of 1 month (range, 0-8 months). Early local control was achieved with $87.5 \%$ success (35/40 patients), local progression being observed in the treatment of bone lesions for three patients and seminal vesicles for two patients.

Considering solely the 31 patients treated in the castrationsensitive setting with exclusive focal therapy (no concomitant ADT), a PSA response was observed in $45.2 \%$ patients $(n=14)$ : partial in $32.3 \%(n=10)$, and complete in $12.9 \%(n=4)$. A visual representation of the PSA response can be found in Figure 3. With a median follow-up of 30 months (IQR 13.3-56.8), the median PFS was 9 months (95\% CI, 6-17), and the median time to initiation of ADT was 11 months (95\% CI, 9-47). Three deaths were observed at the end of follow-up (at respectively $30-$, 60- and 85-months post-procedure).

In this population, comparing patients presenting a postprocedure PSA response (at least partial) and those who did not, a significant difference was found regarding the PFS with median survivals of 17 and 8 months respectively ( $p=0.002$, Figure 4), 


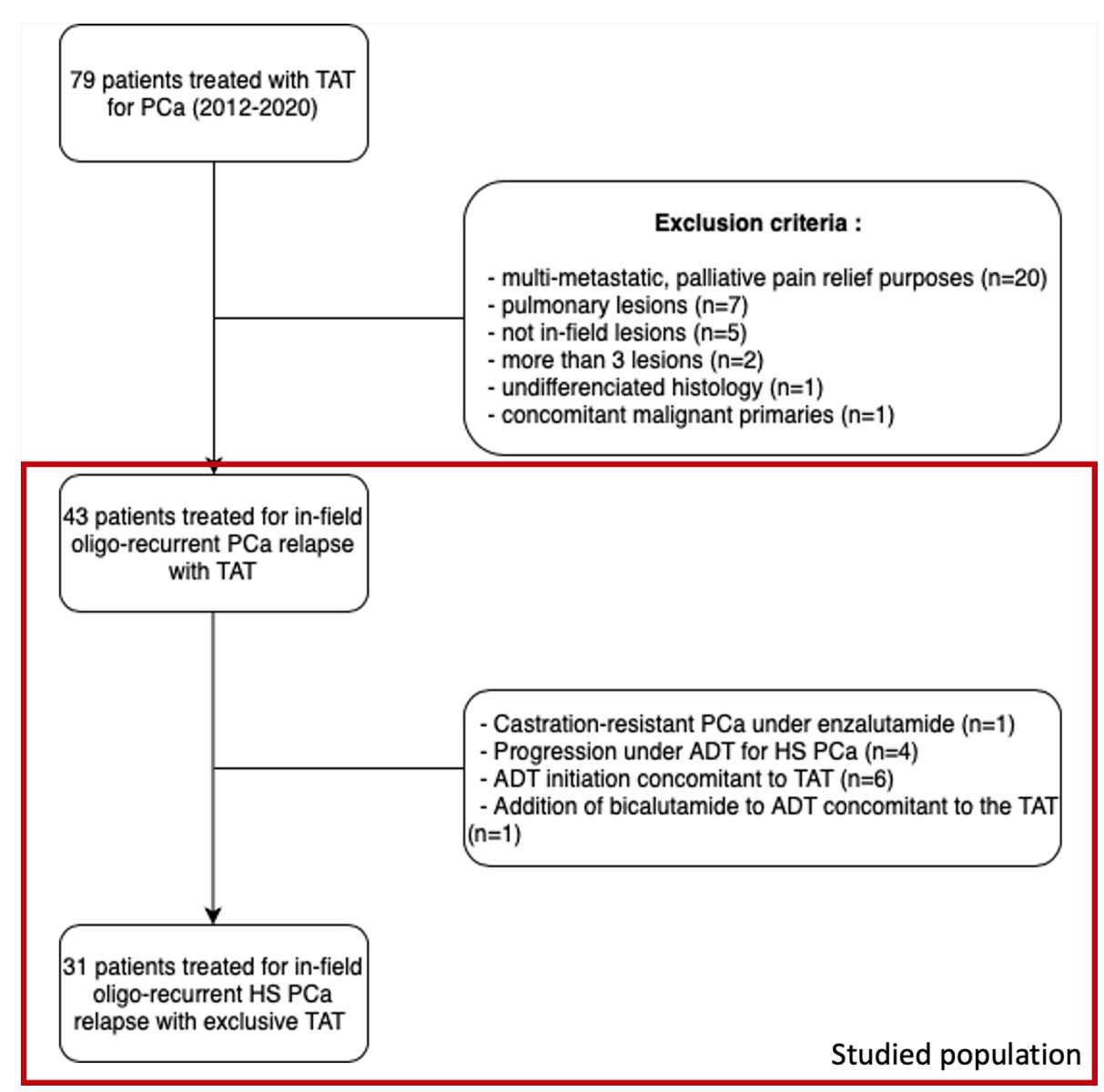

FIGURE 2 | Flowchart of patient selection. TAT, thermo-ablative therapy, PCa, prostate cancer, ADT, androgen deprivation therapy, HS, hormone-sensitive.

and time to ADT initiation (21 versus 8 months, $\mathrm{p}=0.014$ ). There was no significant difference found in terms of initial tumoral characteristics, extension, or pre-procedure data, notably regarding the pre-procedure PSA (means of 4.24 and $4.27 \mathrm{ng} / \mathrm{ml}$ respectively, $\mathrm{p}=0.77$ ) and PSA-DT (10.5 vs 9.9 months respectively, $\mathrm{p}=0.46$ ) (Table 2). Among the four patients who displayed complete PSA response at the first follow-up, prolonged responses were obtained: no event at 7 and 73 months of follow-up for two patients, biochemical relapse at 16 months and 45 months requiring $\mathrm{ADT}$ initiation for the two others.

Following thermo-ablative therapy, radiological patterns of progression among the 26 patients experiencing biochemical recurrence favored single site recurrences (54\%). Multisite relapse ( $\geq 5$ lesions) was observed in $10 \%$. In patients treated for bone lesions, the vast majority ( $83 \%$ ) experienced recurrences that included an osseous site, and for relapses after treating nodal sites, $71.4 \%$ occurred exclusively to nodal sites, and $28.6 \%$ showcased osseous lesions. Local relapses were observed in six patients $(19.4 \%)$, mostly treated on the prostate post-operative bed $(66.7 \%)$. Finally, in five cases, no radiological target could be identified despite reaching biochemical levels of recurrence.

\section{Toxicity Assessment}

Six toxicity events were observed in five patients (11.6\%) classified according to the Dindo-Clavien classification: four grade I (acute urinary tract burning sensation, acute dysuria for one week, acute post-operative local pain, prolonged S1 sciatalgia), one grade IIIa (acute urinary retention due to an uretral stenosis requiring a catheter), and one grade IIIb event (urinary incontinence and uretral stenosis requiring endoscopic uretrotomy 16 months after a cryotherapy procedure for a local prostatic recurrence post-RT). Both grade III events occurred after procedures targeting the prostate, with per-operative difficulties due to local considerations (small prostatic gland, fibrosis due to previous RT +/- highintensity focused ultrasound treatments) and already harboring pre-procedure obstructive urinary symptoms. We did not identify any post-operative urinary or digestive fistula.

\section{DISCUSSION}

In our study, we showed that thermo-ablative therapies were safe, with around 45\% PSA responses when treating in-field ADT-free oligo-recurrent PCa, allowing a median of 21-month systemic treatment deferral for PSA responders. 
TABLE 1 | Baseline patient characteristics among prostate cancer patients treated by thermo-ablative therapy for in-field oligo-recurrence.

\begin{tabular}{|c|c|}
\hline & $\begin{array}{l}\text { Overall cohort } \\
\qquad(n=43)\end{array}$ \\
\hline Age at procedure (mean [extremes], years) $(n=43)$ & $72[51 ; 86]$ \\
\hline \multicolumn{2}{|l|}{ Gleason score $(n=42)$} \\
\hline 5 & $1(2.4 \%)$ \\
\hline 6 & $4(9.6 \%)$ \\
\hline 7 & $24(57.1 \%)$ \\
\hline $3+4$ & $13(30.9 \%)$ \\
\hline $4+3$ & $11(26.2 \%)$ \\
\hline 8 & $8(19.0 \%)$ \\
\hline $3+5$ & $2(4.7 \%)$ \\
\hline $4+4$ & $6(14.3 \%)$ \\
\hline 9 & $5(11.9 \%)$ \\
\hline \multicolumn{2}{|l|}{ ISUP score $(n=42)$} \\
\hline 1 & $5(11.9 \%)$ \\
\hline 2 & $13(31.0 \%)$ \\
\hline 3 & $11(26.1 \%)$ \\
\hline 4 & $6(14.3 \%)$ \\
\hline 5 & 7 (16.7\%) \\
\hline Initial PSA [mean (extremes), ng/ml] ( $\mathrm{n}=32)$ & $16.9[3.86 ; 129]$ \\
\hline \multicolumn{2}{|l|}{ Initial T stage $(n=42)$} \\
\hline $\mathrm{T} 2 \mathrm{a}$ & $1(2.4 \%)$ \\
\hline $\mathrm{T} 2 \mathrm{~b}$ & $6(14.3 \%)$ \\
\hline $\mathrm{T} 2 \mathrm{c}$ & $8(19.0 \%)$ \\
\hline T3a & $13(31.0 \%)$ \\
\hline $\mathrm{T} 3 \mathrm{~b}$ & 14 (33.3\%) \\
\hline \multicolumn{2}{|l|}{ Initial N stage ( $\mathrm{n}=42)$} \\
\hline NO & 39 (92.9\%) \\
\hline N1 & $3(7.1 \%)$ \\
\hline \multicolumn{2}{|l|}{ Initial R stage (in case of surgery) ( $n=31$ ) } \\
\hline RO & $23(74.2 \%)$ \\
\hline $\mathrm{R} 1$ & 8 (25.8\%) \\
\hline \multicolumn{2}{|l|}{ Treatment sequence $(n=43)$} \\
\hline Initial prostatectomy + salvage RT & $32(74.4 \%)$ \\
\hline Extended lymphadenectomy & $21(65.6 \%)$ \\
\hline Limited lymphadenectomy & 2 (6.3\%) \\
\hline No lymphadenectomy & $9(28.1 \%)$ \\
\hline Initial RT +/- ADT & $11(25.6 \%)$ \\
\hline \multicolumn{2}{|l|}{ Previous RT field ( $n=43$ ) } \\
\hline Prostate only & $1(2.3 \%)$ \\
\hline Prostate + whole-pelvis & $10(23.3 \%)$ \\
\hline Prostatic bed only & $12(27.9 \%)$ \\
\hline Prostatic bed + whole-pelvis & 20 (46.5\%) \\
\hline Last PSA before procedure [mean (extremes), ng/ml] ( $n=43)$ & $5.0[0.3 ; 22.0]$ \\
\hline $\begin{array}{l}\text { Calculated PSA doubling time [mean (extremes), months] } \\
(\mathrm{n}=43)\end{array}$ & $8.9[1.4 ; 55.1]$ \\
\hline Target maximum diameter [mean (extremes), mm] $(n=43)$ & $15.2[6 ; 57]$ \\
\hline \multicolumn{2}{|l|}{ Type of lesion treated $(n=49)$} \\
\hline Bone & $13(26.5 \%)$ \\
\hline Node & $15(30.6 \%)$ \\
\hline Prostate, prostatic bed or seminal vesicles & $21(42.9 \%)$ \\
\hline \multicolumn{2}{|l|}{ Concomitant ADT $(n=43)$} \\
\hline No & $31(72.1 \%)$ \\
\hline Yes, started or modified concomitantly & 7 (16.3\%) \\
\hline Yes, previously in place & $5(11.6 \%)$ \\
\hline HSPC & $4(9.3 \%)$ \\
\hline HRPC & $1(2.3 \%)$ \\
\hline
\end{tabular}

$R T$, radiation therapy; $A D T$, androgen deprivation therapy; $P S A$, prostate serum antigen; HSPC, hormone-sensitive prostate cancer; HRPC, hormone-resistant prostate cancer.

Patterns of PCa recurrence after initial local therapy have been shown to be associated with prostatic cancer-specific survival (PCSS). In 2,694 patients treated with prostate-only RT, prostate was the most common first recurrence site in the low, intermediate, and high risk groups with an 8-year cumulative incidence of 3.5, 9.8, and $14.6 \%$ respectively. Moreover, in the 474 patients with clinically detected recurrence, the most common first recurrence site was local in $55.3 \%$, bone in $33.5 \%$, pelvic lymph nodes in $21.3 \%$, and abdominal lymph nodes in 9.1\% (30). Likewise, in 574 men treated by salvage RT (SRT) after prostatectomy between 1986 and 2013, the 8-year rates of local, regional, and distant failure were 2, 6 , and $21 \%$ respectively, of which $17 \%$ were lymphotrophic, $50 \%$ osteotrophic, and $31 \%$ multifocal, a repartition prognostic for distant metastases-free survival and PCSS (31). In our cohort, similarly to Deek et al. (32), patterns of recurrence after MDT were mostly oligo-progressions, with only three patients showing multisite recurrence of $\geq 5$ metastases. Recurrence tended to occur in osseous sites after treating bone targets, and nodal or bone locations when treating patients with nodal-only dissemination.

In case of local relapse after RT, a meta-analysis was recently published including 150 studies regarding outcomes of local therapies. Adjusted 5-year recurrence-free survival ranged from $50 \%$ after cryotherapy to $60 \%$ after high-dose-rate brachytherapy and SBRT, with no significant differences between any modality and radical prostatectomy. Severe GU toxicity was however significantly lower with salvage RT or cryotherapy than with salvage RP (33). Data for salvage prostate SBRT re-irradiation are emerging but the technique must be administered with caution in expert centers and highly selected patients, with 2- and 3-year disease-free survival rates ranging from 40 to $82 \%$ among 38 studies; inclusion in clinical trials is recommended (34).

Thanks to the advent of new radiotracers like Choline or PSMA, oligo-metastatic states (either synchronous or metachronous) are increasingly discovered. In 9,632 restaging Choline PET/CT performed between 2007 and 2015 for biochemical relapse post-RP or RT, Graziani et al. found an incidence of $37.7 \%$ of oligometastatic disease defined as one to three lesions (35). These new imaging modalities are especially effective for PSA rates $<20 \mathrm{ng} / \mathrm{ml}$ compared to the traditional triquetra of CT, bone scintigraphy and MRI. Although CholinePET already offers good detection, its sensitivity is highly dependent on PSA levels and kinetics (36). PSMA-PET offers even better sensitivity, with more detected lesions in low PSA patients and offering higher contrast with the background noise $(37,38)$. This is raising new discussions, as this ability to detect and potentially treat locally a few small hypermetabolic lesions questions its place in the oncological strategy. It is hypothesized that local treatments might reduce the number of circulating tumor cells, which were shown to be associated with an increased risk of progression and mortality (39).

Several ongoing or recently published trials explore outcomes of MDT in this oligo-metastatic setting, either nodal pelvic or distant metastatic. For instance, in the phase II STOMP trial, MDT to up to three extracranial oligo-recurrent lesions visible on Choline-PET tended to increase the ADT-free survival compared to surveillance ( 8 vs $34 \%$ at 5 years, $\mathrm{p}=0.06$ ) (12). Nevertheless, there is significant heterogeneity between local treatment protocols (SBRT fractionation and dose, surgical procedure) and diagnostic imaging between these trials. 


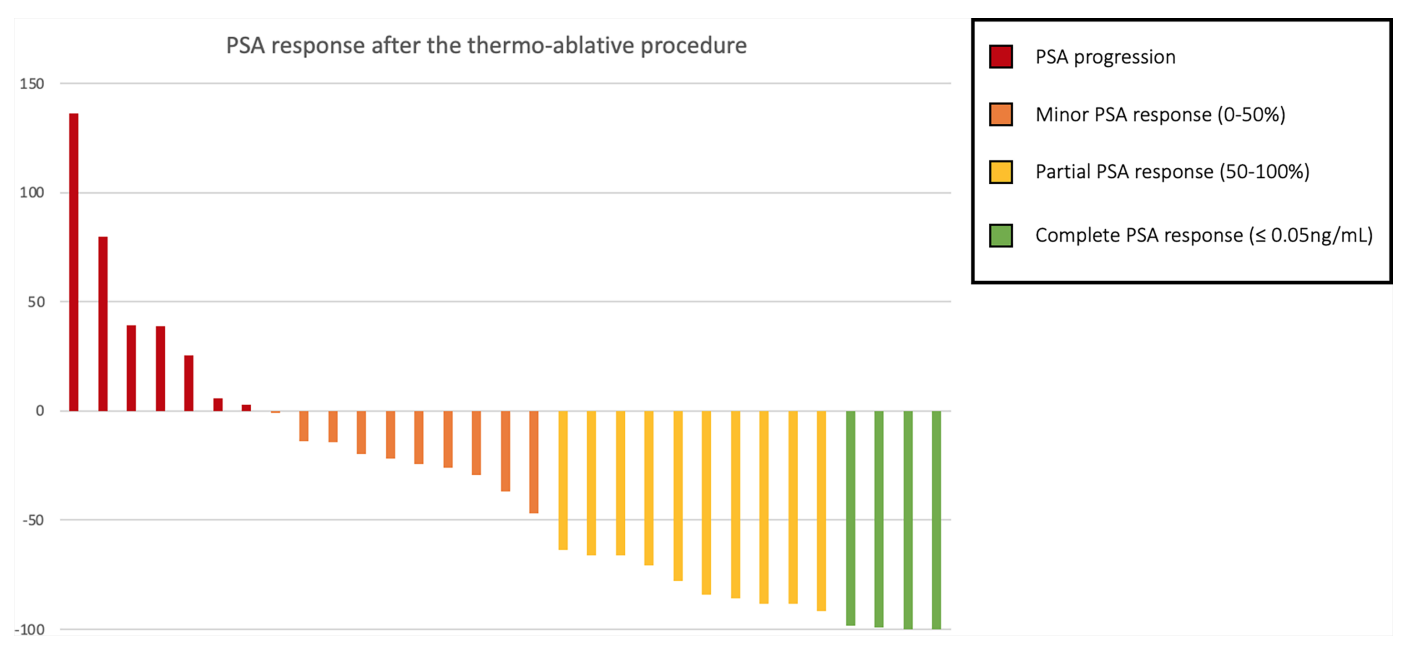

FIGURE 3 | PSA response in percent of pre-procedure PSA among oligo-recurrent hormone-sensitive prostate cancer patients treated with exclusive thermo-ablative therapy.

Inclusions can also be difficult, mostly due to an unwillingness to be assigned in a treatment arm based on randomization (40).

A limited number of papers have been published about infield MDT after a prior RT, and series comprising re-irradiated patients often intertwine with RT-naïve patients $(23,41)$. In our cohort of hormone-sensitive PCa oligo-recurrent patients, PSA response was obtained in $45.2 \%$ of patients without concomitant ADT, postponing in responders with a median delay of 17 months the need of ADT initiation, disease recurrence or death (9 months in the overall population).

This MDT strategy could also be discussed in an intensification strategy concomitant to ADT. For instance, Kroeze et al. found in 305 PSMA PET-positive oligo-recurrent patients that MDT + ADT significantly improved the biological PFS (hazard ratio $0.28,95 \%$ confidence interval $0.16-0.51$ ), but was not significantly different between MDT and $\leq 6$ months of

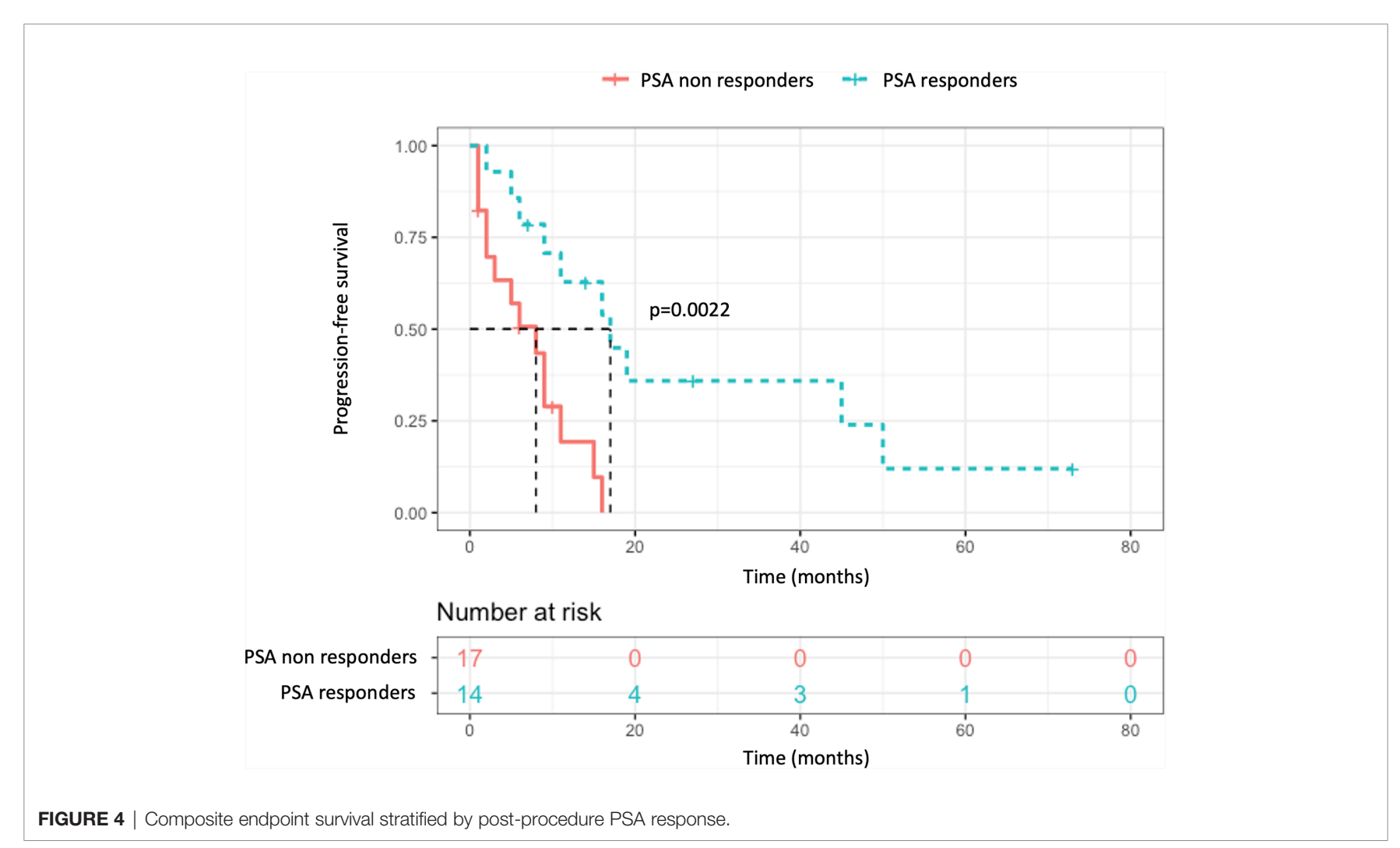


TABLE 2 | Statistical comparison of initial and pre-procedure data between PSA responders and non-responders.

\begin{tabular}{lccc}
\hline & $\begin{array}{c}\text { PSA } \\
\text { responders }\end{array}$ & $\begin{array}{c}\text { PSA non } \\
\text { responders }\end{array}$ & p-value \\
\hline Age at procedure (years, mean) & 69.1 & 71.0 & 0.46 \\
PSA at diagnosis (ng/ml, mean) & 14.6 & 11.5 & 0.34 \\
Last PSA pre-TAT (ng/ml, mean) & 4.24 & 4.27 & 0.77 \\
PSA doubling time (months, mean) & 10.49 & 9.85 & 0.46 \\
Initial Gleason score & & & 0.16 \\
Initial ISUP group & & & 0.45 \\
Initial T-stage & & & 0.15 \\
Initial N-stage & & & 0.48 \\
Initial D'Amico risk group & & & 0.77 \\
Number of lesions treated & & & \\
\hline
\end{tabular}

TAT, thermo-ablative therapy.

ADT or MDT alone $(\mathrm{p}=0.121)$. However, the risk of disease progression was higher after local radiotherapy alone without immediate ADT (42) and consequently the need for further treatments. Combination with novel agents like durvalumab, an anti-PD-L1, may also bear promises, like in the ongoing POSTCARD trial (NCT03795207).

Finally, MDT could also be debated for castration-resistant $\mathrm{PCa}$, even if our population was limited in this regard (43). For example, in a prospective study of 29 patients (of which $37.9 \%$ were castrationresistant) with oligo-metastatic PCa, SBRT allowed controlled PSA levels in 20 patients (with a median follow-up of 11.5 months), thus avoiding the use of systemic therapy and delayed its use by a median of 39.7 months in the remaining nine patients (44). In this castrationresistant setting a few trials are underway, like the FORCE trial (NCT03556904) for oligo-metastatic patients or the STEREO-REPRO trial (NCT03438552) now in phase I to determine the optimal SBRT fractionation before entering phase II aiming to estimate the efficacy of repeat salvage SBRT in terms of biochemical relapse-free survival rate for intraprostatic tumor recurrence.

The choice of focal treatment modality in our center was mainly motivated by the high experience of our interventional radiologists, and the easy access to thermo-ablative techniques, compared to SBRT which was not available at the time in our institution. Selecting the optimal technique should be discussed on a patient-basis, as well as according to local habits.

In our cohort, tolerance was acceptable with only two grade $\geq$ III events according to Dindo-Clavien, both in patients treated on the prostate with prior obstructive urinary symptoms and locally contributing factors. Risks are mainly dependent on the treated target: nervous and vascular lesions for extra-prostatic procedures (especially lymph nodes, with a risk of obturator and sciatic nerves injury when dealing with lesions close to the obturator ring/ presacral spaces); genito-urinary and sexual complications in case of local relapse, which is similarly reported in the available literature ( $0-31 \%$ rates of erectile dysfunction, $1-17 \%$ rates of urinary retention, and less than 5\% rates of urinary incontinence) (45-48).

Our study holds several limitations mostly due to its retrospective nature and the limited size of our cohort emanating from a single institution and containing heterogeneous patients in terms of initial tumor characteristics, natural history, and previous treatments. In the final analysis, only ADT-free hormone-sensitive patients were described, as concomitant systemic treatment would have affected the outcomes. Due to the extended period of study, there is also a discrepancy regarding imaging modalities, notably with the recent advent of Choline and PSMA tracers, which could have impacted the therapeutic strategy.

In the end, patient selection was crucial to sort out which patients could benefit the most from these aggressive strategies. Undergoing trials should provide an answer whether focal therapy is beneficial when treating in-field oligo recurrent patients and eventually translates to time-spared, or time-wasted. In our cohort, no factor (initial patient and tumor's characteristics or pre-procedure PSA rates and kinetics) was found to be associated with the PSA response. Associating the PSA-DT, biopsy Gleason score and interval from primary therapy to biochemical failure could further stratify patients according to recent recommendations, notably to select patients benefiting from early ADT initiation after non-metastatic PCa relapse $(49,50)$. In this matter, advances in PCa genomics or even radiomics and artificial intelligence could also generate new hopes with regard to personalized medicine (51-53). Tumor mutational profiles, such as driver mutations in TP53 or alterations in other tumor suppressor genes, could be associated with disparate outcomes among oligo-metastatic PCa, possibly identifying in the near future patients with aggressive features who may benefit from intensified treatment (54-56).

\section{CONCLUSION}

Thermo-ablative procedures for in-field oligo-recurrent PCa are a feasible option in terms of local control and biochemical response, possibly allowing systemic treatment deferral for patients with in-field oligo-recurring PCa or even potentiating its effects. Patient selection is crucial and could benefit from advances in imaging and prognostic markers. Given the risk of morbidity and need of technical experience, these procedures should be discussed on a case-by-case basis in a multidisciplinary setting and preferably performed in expert centers.

\section{DATA AVAILABILITY STATEMENT}

The raw data supporting the conclusions of this article can be made available by the authors, upon request.

\section{ETHICS STATEMENT}

The studies involving human participants were reviewed and approved by Collège de Recherche, Institut Bergonié (April 12, 2021). Written informed consent for participation was not required for this study in accordance with the national legislation and the institutional requirements.

\section{AUTHOR CONTRIBUTIONS}

NG and N-SV: data collection. NG: data processing and statistical analysis, and original draft writing. XB, RG, VC, JP, GR, and PS: data collection. XB, GR, and PS: supervision. NG, XB, N-SV, RG, A-LC, VC, JP, GR, and PS: manuscript correction. All authors contributed to the article and approved the submitted version. 


\section{REFERENCES}

1. Ferlay J, Ervik M, Lam F, Colombet M, Mery L, Piñeros M, et al. Global Cancer Observatory: Cancer Today. Lyon, France: International Agency for Research on Cancer (2018). Available at: https://gco.iarc.fr/today.

2. Parker CC, James ND, Brawley CD, Clarke NW, Hoyle AP, Ali A, et al. Radiotherapy to the Primary Tumour for Newly Diagnosed, Metastatic Prostate Cancer (STAMPEDE): A Randomised Controlled Phase 3 Trial. Lancet (2018) 392(10162):2353 66. doi: 10.1016/S0140-6736(18)32486-3

3. Boevé LMS, Hulshof MCCM, Vis AN, Zwinderman AH, Twisk JWR, Witjes WPJ, et al. Effect on Survival of Androgen Deprivation Therapy Alone Compared to Androgen Deprivation Therapy Combined With Concurrent Radiation Therapy to the Prostate in Patients With Primary Bone Metastatic Prostate Cancer in a Prospective Randomised Clinical Trial: Data From the HORRAD Trial. Eur Urol (2019) 75(3):410 8. doi: 10.1016/j.eururo.2018.11.008

4. Hellman S, Weichselbaum RR. Oligometastases. J Clin Oncol (1995) 13(1):810. doi: 10.1200/JCO.1995.13.1.8

5. Szturz P, Nevens D, Vermorken JB. Oligometastatic Disease Management: Finding the Sweet Spot. Front Oncol (2020) 10:617793. doi: 10.3389/ fonc. 2020.617793

6. Tosoian JJ, Gorin MA, Ross AE, Pienta KJ, Tran PT, Schaeffer EM. Oligometastatic Prostate Cancer: Definitions, Clinical Outcomes, and Treatment Considerations. Nat Rev Urol (2017) 14(1):15 25. doi: 10.1038/nrurol.2016.175

7. Iyengar P, Wardak Z, Gerber DE, Tumati V, Ahn C, Hughes RS, et al. Consolidative Radiotherapy for Limited Metastatic Non-Small-Cell Lung Cancer: A Phase 2 Randomized Clinical Trial. JAMA Oncol (2018) 4(1): e173501. doi: 10.1001/jamaoncol.2017.3501

8. Palma DA, Olson R, Harrow S, Gaede S, Louie AV, Haasbeek C, et al. Stereotactic Ablative Radiotherapy Versus Standard of Care Palliative Treatment in Patients With Oligometastatic Cancers (SABR-COMET): A Randomised, Phase 2, Open-Label Trial. Lancet (2019) 393(10185):2051 8. doi: 10.1016/S0140-6736(18)32487-5

9. Gomez DR, Tang C, Zhang J, Blumenschein GR, Hernandez M, Lee JJ, et al. Local Consolidative Therapy Vs. Maintenance Therapy or Observation for Patients With Oligometastatic Non-Small-Cell Lung Cancer: Long-Term Results of a Multi-Institutional, Phase II, Randomized Study. JCO (2019) 37 (18):1558 65. doi: 10.1200/JCO.19.00201

10. Bernard B, Gershman B, Karnes RJ, Sweeney CJ, Vapiwala N. Approach to Oligometastatic Prostate Cancer. Am Soc Clin Oncol Educ Book (2016) 36):119 29. doi: 10.1200/EDBK_159241

11. Supiot S, Pasquier D, Buthaud X, Magné N, Beckendorf V, Sargos P, et al. Oligopelvis-GETUG P07: A Multicenter Phase II Trial of Combined Salvage Radiotherapy and Hormone Therapy in Oligorecurrent Pelvic Node Relapses of Prostate Cancer. JCO (2020) 38(6_suppl):93. doi: 10.1200/ JCO.2020.38.6_suppl.93

12. Ost P, Reynders D, Decaestecker K, Fonteyne V, Lumen N, De Bruycker A, et al. Surveillance or Metastasis-Directed Therapy for Oligometastatic Prostate Cancer Recurrence (STOMP): Five-Year Results of a Randomized Phase II Trial. JCO (2020) 38(6_suppl):10. doi: 10.1200/JCO.2017.75.4853

13. Siva S, Bressel M, Murphy DG, Shaw M, Chander S, Violet J, et al. Stereotactic Abative Body Radiotherapy (SABR) for Oligometastatic Prostate Cancer: A Prospective Clinical Trial. Eur Urol (2018) 74(4):455 62. doi: 10.1016/ j.eururo.2018.06.004

14. Supiot S, Libois V, Guimas V, Rio E, Rolland F, Bompas E, et al. Prostate Cancer With Oligometastatic Relapse: Combining Stereotactic Ablative Radiotherapy and Durvalumab, a Randomized Phase II Trial (POSTCARD - GETUG-P13). JCO (2019) 37(15_suppl):TPS5088. doi: 10.1200/ JCO.2019.37.15_suppl.TPS5088

15. Phillips R, Shi WY, Deek M, Radwan N, Lim SJ, Antonarakis ES, et al. Outcomes of Observation vs Stereotactic Ablative Radiation for Oligometastatic Prostate Cancer: The ORIOLE Phase 2 Randomized Clinical Trial. JAMA Oncol (2020) 6 (5):650. doi: 10.1001/jamaoncol.2020.0147

16. Blanchard P, Foulon S, Louvel G, Habibian M, Fizazi K. Rôle De La Radiothérapie Ablative Des Métastases Chez Les Patients Atteints De Cancer De La Prostate Oligométastatique Hormonosensible: Un Essai Du Groupe D'étude Des Tumeurs Urogénitales Et De L'Association Française D’urologie. Cancer/Radiothérapie (2017) 21(6 7):491 4. doi: 10.1016/j.canrad.2017.06.007
17. Boehmer D, Maingon P, Poortmans P, Baron M-H, Miralbell R, Remouchamps V, et al. Guidelines for Primary Radiotherapy of Patients With Prostate Cancer. Radiother Oncol (2006) 79(3):259-69. doi: 10.1016/j.radonc.2006.05.012

18. Lawton CA, Michalski J, El-Naqa I, Buyyounouski MK, Lee WR, Menard C, et al. RTOG GU Radiation Oncology Specialists Reach Consensus on Pelvic Lymph Node Volumes for High-Risk Prostate Cancer. Int J Radiat Oncol Biol Phys (2009) 74(2):383-7. doi: 10.1016/j.ijrobp.2008.08.002

19. Poortmans P, Bossi A, Vandeputte K, Bosset M, Miralbell R, Maingon P, et al. Guidelines for Target Volume Definition in Post-Operative Radiotherapy for Prostate Cancer, on Behalf of the EORTC Radiation Oncology Group. Radiother Oncol (2007) 84(2):121-7. doi: 10.1016/j.radonc.2007.07.017

20. Lestingi JFP, Guglielmetti GB, Trinh QD, Coelho RF, Pontes JJr, Bastos DA, et al. Extended Versus Limited Pelvic Lymph Node Dissection During Radical Prostatectomy for Intermediate- and High-Risk Prostate Cancer: Early Oncological Outcomes From a Randomized Phase 3 Trial. Eur Urol (2021) 79(5):595-604. doi: 10.1016/j.eururo.2020.11.040

21. Pound CR. Natural History of Progression After PSA Elevation Following Radical Prostatectomy. JAMA (1999) 281(17):1591. doi: 10.1001/jama.281.17.1591

22. Tsoumakidou G, Buy X, Garnon J, Enescu J, Gangi A. Percutaneous Thermal Ablation: How to Protect the Surrounding Organs. Techniques Vasc Interventional Radiol (2011) 14(3):170 6. doi: 10.1053/j.tvir.2011.02.009

23. Glicksman RM, Metser U, Vines D, Valliant J, Liu Z, Chung PW, et al. Curative-Intent Metastasis-Directed Therapies for Molecularly-Defined Oligorecurrent Prostate Cancer: A Prospective Phase II Trial Testing the Oligometastasis Hypothesis. Eur Urol (2021) S0302283821001512. doi: 10.1016/j.eururo.2021.02.031

24. Petrylak DP, Ankerst DP, Jiang CS, Tangen CM, Hussain MHA, Lara PN, et al. Evaluation of Prostate-Specific Antigen Declines for Surrogacy in Patients Treated on SWOG 99-16. JNCI: J Natl Cancer Institute (2006) 98 (8):516 21. doi: $10.1093 /$ inci/dji129

25. Eisenhauer EA, Therasse P, Bogaerts J, Schwartz LH, Sargent D, Ford R, et al. New Response Evaluation Criteria in Solid Tumours: Revised RECIST Guideline (Version 1.1). Eur J Cancer (2009) 45(2):228-47. doi: 10.1016/ j.ejca.2008.10.026

26. Roach M, Hanks G, Thames H, Schellhammer P, Shipley WU, Sokol GH, et al. Defining Biochemical Failure Following Radiotherapy With or Without Hormonal Therapy in Men With Clinically Localized Prostate Cancer: Recommendations of the RTOG-ASTRO Phoenix Consensus Conference. Int $J$ Radiat Oncol Biol Physics (2006) 65(4):965 74. doi: 10.1016/j.ijrobp.2006.04.029

27. ASTRO. Consensus Statement: Guidelines for PSA Following Radiation Therapy. Int J Radiat Oncol Biol Physics (1997) 37(5). S0360301697000023. doi: 10.1016/S0360-3016(97)00002-3

28. Dindo D, Demartines N, Clavien P-A. Classification of Surgical Complications: A New Proposal With Evaluation in a Cohort of 6336 Patients and Results of a Survey. Ann Surgery (2004) 240(2):205 13. doi: 10.1097/01.sla.0000133083.54934.ae

29. Scher HI, Morris MJ, Stadler WM, Higano CS, Halabi S, Smith MR, et al. The Prostate Cancer Working Group 3 (PCWG3) Consensus for Trials in Castration-Resistant Prostate Cancer (CRPC). JCO (2015) 33(15_suppl). 5000. doi: 10.1200/jco.2015.33.15_suppl.5000

30. Zumsteg ZS, Spratt DE, Romesser PB, Pei X, Zhang Z, Kollmeier M, et al. Anatomical Patterns of Recurrence Following Biochemical Relapse in the Dose Escalation Era of External Beam Radiotherapy for Prostate Cancer. J Urol (2015) 194(6):1624 30. doi: 10.1016/j.juro.2015.06.100

31. Jackson WC, Desai NB, Abugharib AE, Tumati V, Dess RT, Lee JY, et al. Anatomical Patterns of Recurrence Following Biochemical Relapse After Post-Prostatectomy Salvage Radiation Therapy: A Multi-Institutional Study. BJU Int (2017) 120(3):351 7. doi: 10.1111/bju.13792

32. Deek MP, Taparra K, Dao D, Chan L, Phillips R, Gao RW, et al. Patterns of Recurrence and Modes of Progression After Metastasis-Directed Therapy in Oligometastatic Castration-Sensitive Prostate Cancer. Int J Radiat Oncol Biol Phys (2021) 109(2):387-95. doi: 10.1016/j.ijrobp.2020.08.030

33. Valle LF, Lehrer EJ, Markovic D, Elashoff D, Levin-Epstein R, Karnes RJ, et al. A Systematic Review and Meta-Analysis of Local Salvage Therapies After Radiotherapy for Prostate Cancer (MASTER). Eur Urol (2020) 109(2):387-95.

34. Baty M, Créhange G, Pasquier D, Palard X, Deleuze A, Gnep K, et al. Salvage Reirradiation for Local Prostate Cancer Recurrence After Radiation Therapy. 
For Who? When? How? Cancer/Radiothérapie (2019) 23(6 7):541 58. doi: 10.1016/j.canrad.2019.07.125

35. Graziani T, Ceci F, Castellucci P, Polverari G, Lima GM, Lodi F, et al. 11c-Choline PET/CT for Restaging Prostate Cancer. Results From 4,426 Scans in a Single-Centre Patient Series. Eur J Nucl Med Mol Imaging (2016) 43 (11):1971-9. doi: 10.1007/s00259-016-3428-z

36. Evangelista L, Briganti A, Fanti S, Joniau S, Reske S, Schiavina R, et al. New Clinical Indications for $18 \mathrm{~F} / 11 \mathrm{C}$-Choline, New Tracers for Positron Emission Tomography and a Promising Hybrid Device for Prostate Cancer Staging: A Systematic Review of the Literature. Eur Urol (2016) 70(1):161 75. doi: 10.1016/j.eururo.2016.01.029

37. Afshar-Oromieh A, Holland-Letz T, Giesel FL, Kratochwil C, Mier W, Haufe S, et al. Diagnostic Performance of 68Ga-PSMA-11 (HBED-CC) PET/CT in Patients With Recurrent Prostate Cancer: Evaluation in 1007 Patients. Eur J Nucl Med Mol Imaging (2017) 44(8):1258 68. doi: 10.1007/s00259-017-3711-7

38. Eiber M, Maurer T, Souvatzoglou M, Beer AJ, Ruffani A, Haller B, et al. Evaluation of Hybrid 68ga-PSMA Ligand PET/CT in 248 Patients With Biochemical Recurrence After Radical Prostatectomy. J Nucl Med (2015) 56 (5):668 74. doi: 10.2967/jnumed.115.154153

39. Resel Folkersma L, San José Manso L, Galante Romo I, Moreno Sierra J, Olivier Gómez C. Prognostic Significance of Circulating Tumor Cell Count in Patients With Metastatic Hormone-Sensitive Prostate Cancer. Urology (2012) 80(6):1328 32. doi: 10.1016/j.urology.2012.09.001

40. On behalf of the members of the Cryotherapy in Prostate Cancer (CROP) study team, Salji M, Jones R, Paul J, Birrell F, Dixon-Hughes J, et al. Feasibility Study of a Randomised Controlled Trial to Compare (Deferred) Androgen Deprivation Therapy and Cryotherapy in Men With Localised Radiation-Recurrent Prostate Cancer. Br J Cancer (2014) 111(3):424 9. doi: 10.1038/bjc.2014.316

41. Jereczek-Fossa BA, Fanetti G, Fodor C, Ciardo D, Santoro L, Francia CM, et al. Salvage Stereotactic Body Radiotherapy for Isolated Lymph Node Recurrent Prostate Cancer: Single Institution Series of 94 Consecutive Patients and 124 Lymph Nodes. Clin Genitourinary Cancer (2017) 15(4):e623 32. doi: 10.1016/ j.clgc.2017.01.004

42. Kroeze SGC, Henkenberens C, Schmidt-Hegemann NS, Vogel MME, Kirste S, Becker J, et al. Prostate-Specific Membrane Antigen Positron Emission Tomography-detected Oligorecurrent Prostate Cancer Treated With MetastasesDirected Radiotherapy: Role of Addition and Duration of Androgen Deprivation. Eur Urol Focus (2021) 7(2):309 16. doi: 10.1016/j.euf.2019.08.012

43. Beauval J-B, Loriot Y, Hennequin C, Rozet F, Barthelemy P, Borchiellini D, et al. Loco-Regional Treatment for Castration-Resistant Prostate Cancer: Is There Any Rationale? A Critical Review From the AFU-GETUG. Crit Rev Oncol/Hematol (2018) 122:144 9. doi: 10.1016/j.critrevonc.2017.12.012

44. Pasqualetti F, Panichi M, Sainato A, Matteucci F, Galli L, Cocuzza P, et al. [18f]Choline PET/CT and Stereotactic Body Radiotherapy on Treatment Decision Making of Oligometastatic Prostate Cancer Patients: Preliminary Results. Radiat Oncol Déc (2016) 11(1):9. doi: 10.1186/s13014-016-0586-x

45. Woodrum DA, Kawashima A, Karnes RJ, Davis BJ, Frank I, Engen DE, et al. Magnetic Resonance Imaging-Guided Cryoablation of Recurrent Prostate Cancer After Radical Prostatectomy: Initial Single Institution Experience. Urology (2013) 82(4):870 5. doi: 10.1016/j.urology.2013.06.011

46. van der Poel HG, van den Bergh RCN, Briers E, Cornford P, Govorov A, Henry AM, et al. Focal Therapy in Primary Localised Prostate Cancer: The European Association of Urology Position in 2018. Eur Urol (2018) 74(1):84 91. doi: 10.1016/j.eururo.2018.01.001

47. Mendez MH, Passoni NM, Pow-Sang J, Jones JS, Polascik TJ. Comparison of Outcomes Between Preoperatively Potent Men Treated With Focal Versus
Whole Gland Cryotherapy in a Matched Population. J Endourol (2015) 29 (10):1193 8. doi: 10.1089/end.2014.0881

48. Valerio M, Shah TT, Shah P, Mccartan N, Emberton M, Arya M, et al. Magnetic Resonance Imaging-Transrectal Ultrasound Fusion Focal Cryotherapy of the Prostate: A Prospective Development Study. Urol Oncol: Semin Original Investigations (2017) 35(4):150.e1-7.

49. Van den Broeck T, van den Bergh RCN, Briers E, Cornford P, Cumberbatch M, Tilki D, et al. Biochemical Recurrence in Prostate Cancer: The European Association of Urology Prostate Cancer Guidelines Panel Recommendations. Eur Urol Focus (2020) 6(2):231 4. doi: 10.1016/j.euf.2019.06.004

50. van den Bergh RCN, van Casteren NJ, van den Broeck T, Fordyce ER, Gietzmann WKM, Stewart F, et al. Role of Hormonal Treatment in Prostate Cancer Patients With Nonmetastatic Disease Recurrence After Local Curative Treatment: A Systematic Review. Eur Urol (2016) 69(5):802 20. doi: 10.1016/ j.eururo.2015.11.023

51. van Dessel LF, van Riet J, Smits M, Zhu Y, Hamberg P, van der Heijden MS, et al. The Genomic Landscape of Metastatic Castration-Resistant Prostate Cancers Reveals Multiple Distinct Genotypes With Potential Clinical Impact. Nat Commun (2019) 10(1):5251. doi: 10.1038/s41467-019-13084-7

52. Bourbonne V, Fournier G, Vallières M, Lucia F, Doucet L, Tissot V, et al. External Validation of an MRI-Derived Radiomics Model to Predict Biochemical Recurrence After Surgery for High-Risk Prostate Cancer. Cancers (2020) 12(4):814. doi: 10.3390/cancers12040814

53. Sargos P, Leduc N, Giraud N, Gandaglia G, Roumiguié M, Ploussard G, et al. Deep Neural Networks Outperform the CAPRA Score in Predicting Biochemical Recurrence After Prostatectomy. Front Oncol (2021) 10:607923. doi: 10.3389 /fonc.2020.607923

54. Hamid AA, Gray KP, Shaw G, MacConaill LE, Evan C, Bernard B, et al. Compound Genomic Alterations of TP53, PTEN, and RB1 Tumor Suppressors in Localized and Metastatic Prostate Cancer. Eur Urol (2019) 76(1):89-97. doi: 10.1016/j.eururo.2018.11.045

55. Deek MP, van der Eecken K, Phillips R, Parikh NR, Isaacsson Velho P, Lotan TL, et al. The Mutational Landscape of Metastatic Castration-Sensitive Prostate Cancer: The Spectrum Theory Revisited. Eur Urol (2021) S03022838(20)31026-5. doi: 10.1016/j.eururo.2020.12.040

56. Mateo J, Seed G, Bertan C, Rescigno P, Dolling D, Figueiredo I, et al. Genomics of Lethal Prostate Cancer at Diagnosis and Castration Resistance. J Clin Invest (2020) 130(4):1743-51. doi: 10.1172/JCI132031

Conflict of Interest: The authors declare that the research was conducted in the absence of any commercial or financial relationships that could be construed as a potential conflict of interest.

Publisher's Note: All claims expressed in this article are solely those of the authors and do not necessarily represent those of their affiliated organizations, or those of the publisher, the editors and the reviewers. Any product that may be evaluated in this article, or claim that may be made by its manufacturer, is not guaranteed or endorsed by the publisher.

Copyright $\odot 2021$ Giraud, Buy, Vuong, Gaston, Cazeau, Catena, Palussiere, Roubaud and Sargos. This is an open-access article distributed under the terms of the Creative Commons Attribution License (CC BY). The use, distribution or reproduction in other forums is permitted, provided the original author(s) and the copyright owner(s) are credited and that the original publication in this journal is cited, in accordance with accepted academic practice. No use, distribution or reproduction is permitted which does not comply with these terms. 